\title{
Loss of Linearity and Symmetrisation in Regulatory Networks
}

\author{
Jacques Demongeot ${ }^{1}$, Eric Goles ${ }^{2,3}$, and Sylvain Sené 4,5 \\ 1 UJF-Grenoble, TIMC-IMAG, Faculté de Médecine, 38706 La Tronche cedex, France \\ 2 Universidad Adolfo Ibañez, Peñalolen, Santiago, Chile \\ 3 ISCV, Institute for Complex Systems, Av. Artillería 600B, Cerro Artillería, \\ Valparaíso, Chile \\ 4 Université de Lyon, INSA-Lyon, LIRIS, F-69621, France \\ 5 IXXI, Institut rhône-alpin des systèmes complexes, 5, rue du Vercors, 69007 Lyon,
} France

\begin{abstract}
This article aims at giving some new theoretical properties of threshold Boolean automata networks which are good mathematical objects to model biological regulatory networks. The objective is the emphasis of a necessary condition for which these networks, when they are governed by a non-linear evolution law, are sensitive to the influence of boundary conditions. Then, this paper opens an argued discussion about the notion of "symmetrisability" of regulatory networks which is relevant to understand some specific dynamical behaviours of real biological networks, and shows that this notion allows to explain an important feature of the Arabidopsis thaliana floral morphogenesis model.

Keywords: Stochastic and deterministic regulatory networks; Robustness; Boundary conditions; Symmetrisation.
\end{abstract}

\section{Introduction}

More and more studies have been opened since a decade about the robustness of biological regulatory networks $[1,2]$. This is explained by the fact the robustness against several kinds of perturbations, such as changes of updating modes [3,4] or changes of topology [5], may bring a better understanding of particular phenomena emerging in biology. The purpose of this paper is to show that, sometimes, a theoretical framework allows to obtain some results which are of great interest not only in this theoretical framework but also in applied frameworks. Moreover, we think that studies on real biological regulatory networks, whatever the used mathematical objects are, need a deep theoretical attention. Indeed, if it is possible to dive a real biological network in a specific framework with relevant theoretical tools, we can reasonably expect to obtain some results on inherent properties of this biological network which could not be obtained with an empirical method.

After a brief section presenting the main definitions which will be used in this paper, Section 3 focuses on artificial stochastic regulatory networks represented by square lattices on $\mathbb{Z}^{2}$. It extends some results already obtained $[6,7]$ on the 
impact of variations of boundary conditions on stochastic regulatory networks whose evolution is governed by a non-linear law and presents a necessary condition for which phase transitions can emerge. These results are interesting from both the theoretical and biological points of view. Indeed, biological regulatory networks present in general natural boundaries such as hormones or microRNAs in general inhibiting the expression of some genes inside the network (some cases of activation being also described as in [8]). Section 4 gives an argued discussion about the dynamical behaviour of the well known Mendoza genetic regulatory network for the floral morphogenesis of the plant Arabidopsis thaliana [9] and shows that it is a direct consequence of its underlying threshold Boolean automata network, which can be seen as a symmetric network.

\section{Preliminaries}

Artificial regulatory networks are widely used in systems biology and present remarkable regularities in their architecture due to the evolution $[10,11,12]$, namely similar values for architectural (connectivity) or dynamic features, or occurrence of same interaction motifs [3], i.e., oriented sub-graphs relating their elements (here genes). These similarities cause identical dynamical behaviours, like existence of periodic attractors, called limit cycles due for example to the same internal motif imposing its periodicity to the global network. Biological regulatory networks are less regular than artificial ones (see Figure 1) but they share many common dynamical concepts that we will give first in whole generality.

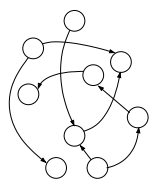

(a)

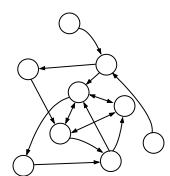

(b)

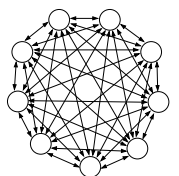

(c)

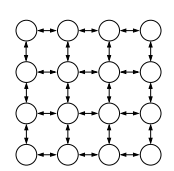

(d)

Fig. 1. Representations of oriented interaction graphs of biological $(a, b)$ and artificial $(c, d)$ regulatory networks.

The interaction matrix $W$ of a regulatory network $R$ of $n$ genes is the oriented and valued incident $n \times n$ matrix of its interaction graph. $W$ is then similar to the synaptic weights matrix, which rules the relationships between neurons in a neural network. The general coefficient $w_{i k}$ of such a matrix $W$ is positive (resp. negative, null) if the gene $k$ activates (resp. inhibits, does not influence) the gene $i$, the state $x_{i}$ of $i$ being equal to 1 (resp. 0), if it is (resp. is not) expressed. In the sequel, we consider two different kinds of regulatory networks, called either deterministic or stochastic. Precisely, in a deterministic regulatory network, the change of state of a gene $i$ between times $t$ and $t+1$ is supposed to obey the 
deterministic transition rule $[13,14]$ :

$$
\begin{gathered}
\forall i \in R, x_{i}(t+1)=H\left(\sum_{j \in \mathcal{N}_{i}} w_{i j} \cdot x_{j}(t)-\theta_{i}\right) \\
\quad \text { or } \\
x(t+1)=H(W \cdot x(t)-\theta)
\end{gathered}
$$

where $H$ is the classical Heaviside sign-step function $(H(x)=0$ if $x<0$ and 1 otherwise), $\mathcal{N}_{i}$ is the set of neighbours of $i$ such that $j \in \mathcal{N}_{i} \Longleftrightarrow w_{i j} \neq 0$ and $\theta_{i}$ is the activation threshold of $i$. In a general way, it is of great biological interest to determine interaction matrices having characteristic properties like: $(i)$ a minimal number of non zero coefficients for a given set of attractors or (ii) a minimal number $P(W)$ of positive loops (i.e. paths on the interaction graph coming from a gene and returning to it after an even number of negative interactions) counted only one time, which controls the number $A(W)$ of attractors $[15,16]$. The connectivity coefficient $K(W)=\frac{I(W)}{n}$ is the mean number of interactions going to a gene, where $I(W)$ is the total number of interactions: $K(W)$ is in general between 1.5 and 3 . About the number $C(W)$ of strong connected components (i.e. with a path between each pair of genes in both senses) containing positive loops, we can conjecture (in particular for feed-forward networks) that:

$$
2 P(W) \geq A(W) \geq 2 C(W) \text { and } A(W) \geq O\left(n^{\frac{1}{2}}\right)
$$

The total number of attractors is, in the general case, of greater order of magnitude, due to the presence of numerous limit cycles both in Hopfield-like (resp. Kauffman-like) random regulatory networks in which the $4^{3}$ different sets of interaction signs $1,-1$ or 0 (resp. the 16 possible sets of Boolean functions) are randomly chosen for the nearest neighbours of any node in the transition rule $[42,43]$.

In the case of stochastic regulatory networks, the introduction of randomness is done by a specific parameter called the temperature, denoted by $T$. By considering the same transition operator as above $R_{x}=H(W x-\theta)$, we compute the probability for the gene $i$ to be in state 1 at time $t+1$ knowing the state of its neighbours at time $t$ :

$$
\forall i \in R, P\left(x_{i}(t+1)=1\right)=\frac{e^{R_{x} / T}}{1+e^{R_{x} / T}}
$$

If $T$ is sufficiently large, this probability is $1 / 2$ (uniform case) and if $T$ is sufficiently small, the stochastic network has the same behaviour as the network with the deterministic transition rule given above. When $T$ is small, we retrieve the deterministic rule. Nevertheless, the real link between the stochastic and the deterministic rules is not actually well known. In this context, we give the following proposition:

Proposition 1. If $T$ is small and $\forall i, j \in \mathbb{Z}^{d}, w_{i i}=u_{0}>\theta, w_{i j}=u_{1}>0$ if $\delta(i, j)=1$, and $w_{i j}=0$ if $\delta(i, j)>1$, where $\delta$ is the L1-distance on $\mathbb{Z}^{d}$, then 
the mean probability $\alpha(T)$ to change from state 1 to state 0 at time $t+1$, with a uniform initial distribution over the $a=\left|\mathcal{N}_{i} \backslash\{i\}\right|$ nodes of $\mathcal{N}_{i} \backslash\{i\}$, is given by:

$$
\alpha(T)=e^{\left(-u_{0}+\theta\right) / T} \cdot\left[\left(1+e^{-u_{1} / T}\right) / 2\right]^{a}
$$

Proof: Let us denote $\mathcal{C}=\left\{x_{i}(t)=1 ; x_{j}(t)=y_{j} ; j \in \mathcal{N}_{i} \backslash\{i\}\right\}$ and develop:

$$
\begin{aligned}
\alpha(T) & =\sum_{y \in \mathcal{N}_{i} \backslash\{i\}} \frac{P\left(x_{i}(t+1)=0 \mid \mathcal{C}\right)}{2^{a}} \\
& \approx \sum_{k=0}^{a}\left(\begin{array}{l}
a \\
k
\end{array}\right) \frac{e^{\left.\left(-u_{0}+\theta\right) / T-k u_{1} / T\right)}}{2^{a}} \\
& =e^{\left(-u_{0}+\theta\right) / T} \cdot\left[\left(1+e^{-u_{1} / T}\right) / 2\right]^{a}
\end{aligned}
$$

Let us note that the value above gives an estimation of the "noise" introduced by the stochastic transition on the deterministic one where the state 0 is in this case never reached.

Before going further, let us give some definitions about the notion of updating modes. An updating mode is: (i) parallel if, at each time step $t$, all the nodes of $R$ are updated synchronously, (ii) sequential if, at each time step, only one node of $R$ is updated, such that all the nodes are updated after $n$ time steps, according to a specific sequence, (iii) block-sequential if, when the network $R$ is divided into disjoint subsets of nodes, the nodes in a same subset execute their local transition function synchronously and the subsets are updated sequentially. Let us remark here that the parallel and the sequential updating modes are particular blocksequential modes. Furthermore, in the context of artificial networks represented by square lattices on $\mathbb{Z}^{2}$, the centre of a regulatory network is the set of minimal eccentricity nodes (the eccentricity $\varepsilon(u)$ of the vertex $u$ of a connected oriented interaction graph $G=(V, E)$ is the maximal distance from $u$ to any other nodes $v$ of $G$ ) and its boundary is simply the set of all geometric boundary nodes of the underlying lattice.

\section{Loss of linearity in stochastic networks}

Some relevant studies $[17,6,7]$ have been recently done on the influence of boundary conditions on two-dimensional square lattices showing both theoretically and by simulation a specific condition under which stochastic threshold Boolean networks are strongly subjected to variations of their boundary conditions. These studies have been based on a specific kind of networks evolution which is characterised by the linear stochastic transition function presented above. Indeed, the networks elements update their state from the time step $t$ to the time step $t+1$ according to two different potentials: $u_{0}$, a function of the auto-interaction weight, and $u_{1}$, a function of the closest neighbours interaction weights. Here, we propose to study two-dimensional square lattices governed by a stochastic law taking also into account a collective potential, denoted by $\phi$, 
which is going to be detailed in the following. Let us remark that the introduction of this coalition potential is translated by a loss of linearity of the stochastic transition function, which makes this kind of problem much more difficult.

Let us first remark that, when time $t$ tends to infinity, the occurrence of configurations is given by an invariant measure $\mu$ on the space of all configurations, which depends on the updating mode of the network. In $[17,7]$ has been given a general expression of $\mu$, for an arbitrary given feed-forward network $R$ of $n$ nodes, for the general block-sequential updating mode. The notion of invariant measure is of particular interest because it allows to define the notion of phase transitions [18]. Indeed, being given a network $R$, a phase transition emerges from the dynamics of $R$, when, for two different fixed boundary conditions $\partial R_{1}$ and $\partial R_{2}$, two different invariant measures $\mu_{\partial R_{1}}$ and $\mu_{\partial R_{2}}$ are computed, i.e. the invariant measure is not unique.

Let us now denote by $\Lambda=R \backslash\{O\}$, where $O$ is one of the central points of the interaction graph $(R, E)$ and define the cylinder $[A, B]$ as follows:

$$
[A, B]=\left\{\sigma \mid \sigma_{i}=1, i \in A ; \sigma_{i}=0, i \in B\right]
$$

The matricial projectivity equation between the probabilities of configurations on $\Lambda$ is defined, as in [17], by the matrix $M$ below:

$$
M=\left(\begin{array}{ccccccc}
1 & 1 & 0 & \ldots & 0 & \ldots & 0 \\
1 & 0 & 1 & \ldots & 0 & \ldots & 0 \\
1 & 0 & 0 & \ldots & 0 & \ldots & 0 \\
\vdots & \vdots & \vdots & \vdots & \vdots & \vdots & \vdots \\
0 & 0 & 0 & \ldots & 0 & \ldots & 1 \\
\Phi_{0} & \Phi_{1} & \Phi_{2} & \ldots & \Phi_{K} & \ldots & \Phi_{2^{d}}
\end{array}\right)
$$

where $\Phi_{0}=\Phi(\Lambda, \emptyset), \Phi_{1}=\Phi(\Lambda \backslash\{1\},\{1\}), \Phi_{2}=\Phi(\Lambda \backslash\{2\},\{2\}), \Phi_{K}=\Phi(\Lambda \backslash K, K)$, and $\Phi_{2 d}=\Phi(\emptyset, \Lambda)$. In this matrix, the first equations represent the classical projectivity: $\forall i \in \Lambda \backslash K, \mu([\Lambda \backslash K, K])+\mu([\Lambda \backslash(K \cup\{i\}), K \cup\{i\}])=\mu([\Lambda \backslash(K \cup$ $\{i\}), K])$, where $\mu([\Lambda \backslash K, K])$ is the probability to observe the configuration $[\Lambda \backslash K, K]$, i.e. the state 1 on $\Lambda \backslash K$ and 0 on $K$. The last equation is just the Bayes formula, i.e. if $\Phi([\Lambda \backslash K, K])$ denotes the probability to observe the state 1 in $O$ knowing the configuration $[\Lambda \backslash K, K]$, we have:

$$
\sum_{K} \Phi(\Lambda \backslash K, K) \cdot \mu([\Lambda \backslash K, K])=\mu([i, \emptyset])
$$

By developing $\operatorname{det} M$ with respect to the last row, we have:

$$
\operatorname{det} M=\sum_{K}(-1)^{|\Lambda \backslash K|} \cdot \Phi(\Lambda \backslash K, K)
$$

where $(-1)^{|\Lambda \backslash K|} \cdot \Phi(\Lambda \backslash K, K)+(-1)^{|K|} \cdot \Phi(K, \Lambda \backslash K)=(-1)^{|K|}(\Phi(\Lambda \backslash K, K)+$ $\Phi(K, \Lambda \backslash K))$, because $|\Lambda|=2 \cdot d$, with:

$$
\Phi(K, \Lambda \backslash K)=\frac{e^{\left(u_{0}+\sum_{i \in K} u_{1, i}+\phi(K)\right) / T}}{1+e^{\left(u_{0}+\sum_{i \in K} u_{1, i}+\phi(K)\right) / T}}
$$


where the potential $\phi$ is symmetrical, i.e. verifies:

$$
\phi(K)=\phi(\Lambda)-\phi(\Lambda \backslash K)=\sum_{\substack{i, j \in K \\ i \neq j}} u_{2, i, j}+\sum_{\substack{i, j, k \in K \\ i \neq j \neq k}} u_{3, i, j, k}
$$

which is the case if the $u_{2, i, j}$ 's (resp. $u_{3, i, j, k}$ 's) are constant and equal to $u_{2}$ (resp. $\left.-u_{2}\right)$.

Proposition 2. If the singleton potential (or external field) $u_{0}$, and the pair, triple and quadruple potentials, respectively denoted by $u_{1, i}$ 's, $u_{2, i, j}$ 's and $u_{3, i, j, k}$ 's, verify:

$$
u_{0}+\sum_{i \in \Lambda} \frac{u_{1, i}}{2}+\sum_{\substack{i, j \in K \\ i \neq j}} \frac{u_{2, i, j}}{2}+\sum_{\substack{i, j, k \in K \\ i \neq j \neq k}} \frac{u_{3, i, j, k}}{2}=0
$$

then $\operatorname{det} M=0$.

Proof: In order to prove that $\operatorname{det} M=0$, it suffices to multiply in the definition formula of $\Phi(K, \Lambda \backslash K)$ the numerator and the denominator by:

$$
1=\frac{e^{\left(-2 u_{0}-\sum_{i \in \Lambda \backslash K} u_{i}-\phi(\Lambda)\right) / T}}{e^{\left(-2 u_{0}-\sum_{i \in \Lambda \backslash K} u_{i}-\phi(\Lambda)\right) / T}}
$$

Then we have:

$$
\begin{aligned}
\Phi(K, \Lambda \backslash K) & =\frac{e^{\frac{-u_{0}-\sum_{i \in \Lambda \backslash K} u_{i}+\phi(K)-\phi(\Lambda)}{T}}}{e^{\frac{-2 u_{0}-\sum_{i \in \Lambda} u_{i}-\phi(\Lambda)}{T}}+e^{\frac{-u_{0}-\sum_{i \in \Lambda \backslash K} u_{i}+\phi(K)-\phi(\Lambda)}{T}}} \\
& =\frac{e^{\frac{-u_{0}-\sum_{i \in \Lambda \backslash K} u_{i}-\phi(\Lambda \backslash K)}{T}}}{1+e^{\frac{-u_{0}-\sum_{i \in \Lambda \backslash K} u_{i}-\phi(\Lambda \backslash K)}{T}}} \\
& =1-\frac{e^{\frac{u_{0}+\sum_{i \in \Lambda \backslash K} u_{i}+\phi(\Lambda \backslash K)}{T}}}{1+e^{\frac{u_{0}+\sum_{i \in \Lambda \backslash K} u_{i}+\phi(\Lambda \backslash K)}{T}}} \\
& =1-\Phi(\Lambda \backslash K, K)
\end{aligned}
$$

Thus, we have $\Phi(\Lambda \backslash K, K)+\Phi(K, \Lambda \backslash K)=1$, and, since we have $|\Lambda|=2 d$, we eventually get: $\operatorname{det} M=\sum_{|K|=0, \ldots, \frac{|\Lambda|}{2}}(-1)^{|K|}=0$.

Let us eventually remark that, if we want to increase the non-linearity of the collective interactions by introducing an interaction of order 4 , namely a quintuple potential denoted by $u_{4, i, j, k, l}$, then, for having $\phi$ symmetrical, we need: $2 \phi(\{i, j\})=2 u_{2}=\phi(\Lambda)=6 u_{2}+4 u_{3}+u_{4}$ and $\phi(\{i, j, k\})=3 u_{2}+u_{3}=\phi(\Lambda)=$ $6 u_{2}+4 u_{3}+u_{4}$, which involves $u_{3}=-u_{2}$ and $u_{4}=0$.

\section{Usefulness of the notion of symmetrisation}

After having focused on theoretical aspects of artificial regulatory networks, it has seemed natural to make a step in the direction of biology and focus on 


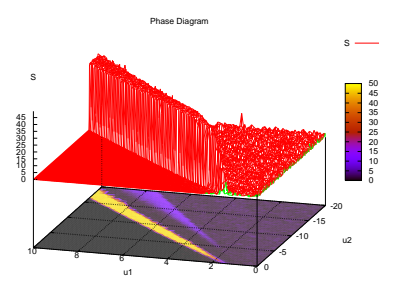

(a)

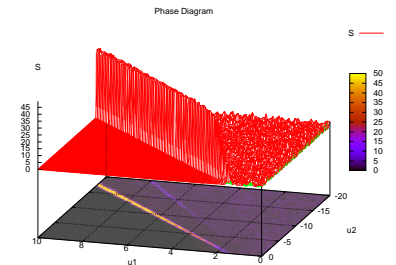

(b)

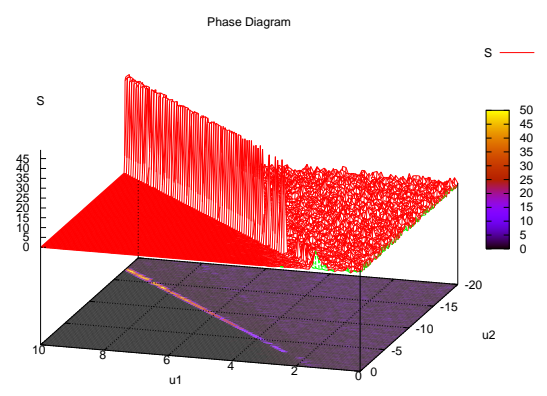

(c)

Fig. 2. Existence of phase transitions for: $(a) 11 \times 11$, (b) $37 \times 37$ and $(c)$ $131 \times 131$ isotropic and translation-invariant attractive networks evolving according to a non linear function with a triple potential $\left(u_{0}=-3,0 \leq u_{1} \leq 10\right)$ on the line $u_{0}+2 u_{1}+u_{2}=0$.

applications relating to real biological genetic networks. So, in this section, we dive into the reality and, by focusing on a well known regulatory network, namely the one of the floral morphogenesis of the plant Arabidopsis thaliana, we propose a bridge, through the notion of symmetrisation which will be explained, allowing to go to and from between these two different levels of abstraction.

\subsection{Elements of literature}

In 1998 has been published an article [9] which presents the dynamics of the genetic regulatory network modelling the floral morphogenesis of the plant Arabidopsis thaliana. This study has been based on the deterministic version of the regulatory networks presented in the introduction and has emphasised that, being given a specific block-sequential updating mode, the temporal evolution of the network can lead its initial configurations to six different fixed points. Among these six fixed points, four correspond to floral tissues (sepal, petal, stamen and carpel), the fifth to an inflorescence tissue and the sixth to a mutant tissue. It is interesting to note that this dynamics holds for all the sequential updating modes. Furthermore, the parallel evolution of this network leads the 
configurations to be attracted not only by these six fixed points but also by seven limit cycles of period two whose biological meaning is not actually known at this time.

This observation is of particular interest because some theoretical results, given by Goles in [19], show that particular deterministic threshold Boolean automata networks get these specific properties, as stated by the two following theorems:

Theorem 1. [20] If the interaction matrix $W$ of a threshold Boolean automata network is symmetric, then the period of the attractors is less or equal to 2.

Theorem 2. [21] If the interaction matrix $W$ of a threshold Boolean automata network is symmetric and such that its diagonal contains only positive coefficients, then the period of the attractors equals 1 for a sequential dynamics.

\subsection{The Mendoza network as a symmetric network}

The purpose of this subsection is to show that the two theorems above can be applied to the Mendoza network (see Figure 3). To do so, it suffices to show that we can find a symmetric network, or more precisely a network $\widetilde{R}$ in which all the dynamical behaviour depends only on specific nodes which belong to symmetric strongly connected components of the underlying interaction graph, leading to the same attractors as the Mendoza network. We will speak thereafter of equivalent networks.

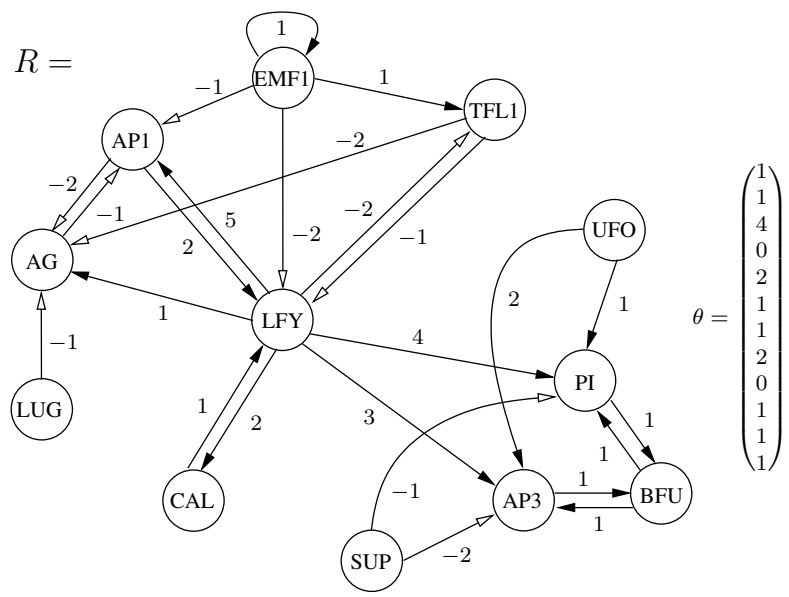

Fig. 3. Genetic regulatory network proposed in [9] by Mendoza and AlvarezBuylla. The interaction weights are written on the graph and the threshold vector is given on the right and is ordered with the following sequence of genes: EMF1, TFL1, LFY, AP1, CAL, LUG, UFO, BFU, AG, AP3, PI et SUP. 
In order to construct such an equivalent symmetric network, we remark first that, in the Mendoza network, the nodes LUG, UFO and SUP are sources of the underlying interaction graph. Thus, they do not depend on themselves or other nodes. Consequently, since their threshold equals 1 , whatever their initial state (at time $t=0$ ) is, they are fixed to 0 for time $t \geq 1$. Formally, we can note : $\forall t \geq 1, \operatorname{LUG}(t)=\operatorname{UFO}(t)=\operatorname{SUP}(t)=H(-1)=0$. Then, let us consider EMF1. Its self-activation makes its state constant, i.e. $\forall t \geq 1, \operatorname{EMF} 1(t)=\operatorname{EMF} 1(0)$. If we consider now LFY, we remark that its state can be equal to 1 only at time $t=0$. Indeed, we can write $\operatorname{LFY}(t+1)=H(-2 \operatorname{EMF} 1(t)-\operatorname{TFL} 1(t)+2 \mathrm{AP} 1(t)+$ $\operatorname{CAL}(t)-4)=0$ since $\forall t,-2 \operatorname{EMF} 1(t)-\operatorname{TFL} 1(t)+2 \operatorname{AP} 1(t)+\operatorname{CAL}(t) \leq 3$. Concerning CAL, we have $\mathrm{CAL}(t+1)=H(2 \mathrm{LFY}(t)-2) \Rightarrow \forall t \geq 1, \mathrm{CAL}(t+1)=$ $H(2 \cdot 0-2)=H(-2)=0$. Thus, $\forall t \geq 2, \operatorname{CAL}(t)=0$. TFL1 depends on the state of EMF1 and LFY. We have TFL1 $(t+1)=H(\operatorname{EMF} 1(t)-2 \mathrm{LFY}-1)$. But we have seen that $\forall t \geq 1, \operatorname{EMF} 1(t)=\operatorname{EMF} 1(0)$ and $\operatorname{LFY}(t)=0$ and, thus, $\forall t, \operatorname{TFL} 1(t)=H(\operatorname{EMF} 1(0)-1)=\operatorname{EMF} 1(0)$. Consequently, these seven genes do not act directly on the dynamics of the network but are only kinds of release mechanisms for the dynamics whose role is stopped, in every case, after time step $t=2$. We are now going to see that the role of AG, AP1, PI, AP3 and BFU is quite more important.

Let us now consider the genes AG and AP1. A first remark is that they depend on each others. Indeed, we have:

$$
\begin{gathered}
\forall t, \operatorname{AG}(t+1)=H(-2 \operatorname{TFL} 1(t)+\operatorname{LFY}(t)-2 \operatorname{AP} 1(t)-\operatorname{LUG}(t)) \\
\Downarrow \\
\forall t \geq 1, \operatorname{AG}(t+1)=H(-2 \operatorname{EMF} 1(0)-2 \operatorname{AP} 1(t))
\end{gathered}
$$

and

$$
\begin{gathered}
\forall t, \operatorname{AP} 1(t+1)=H(-\operatorname{EMF} 1(t)+5 \operatorname{LFY}(t)-\operatorname{AG}(t)) \\
\Downarrow \\
\forall t \geq 1, \operatorname{AP} 1(t+1)=H(-\operatorname{EMF} 1(0)-\operatorname{AG}(t))
\end{gathered}
$$

Since $\forall \lambda>0, H(\lambda u)=H(u)$, we can write:

$$
\begin{aligned}
& \forall t \geq 1, \operatorname{AG}(t+1)=H(-\operatorname{EMF} 1(0)-\operatorname{AP} 1(t)) \\
& \forall t \geq 1, \operatorname{AP} 1(t+1)=H(-\operatorname{EMF} 1(0)-\operatorname{AG}(t))
\end{aligned}
$$

which will be thus represented by a symmetrical strongly connected component in $\widetilde{R}$.

With the same kind of reasoning about the genes AP3, PI and BFU, we obtain:

$$
\begin{gathered}
\forall t \geq 1, \operatorname{AP} 3(t+1)=H(\operatorname{BFU}(t)-1) \\
\forall t \geq 1, \operatorname{PI}(t+1)=H(\operatorname{BFU}(t)-1) \\
\forall t \geq 1, \operatorname{BFU}(t+1)=H(\operatorname{AP} 3(t)+\operatorname{PI}(t)-2)
\end{gathered}
$$


which corresponds to another symmetrical strongly connected component of $\widetilde{R}$. In order to satisfy the constraints exposed above, we can construct $\widetilde{R}$ such that it is presented in Figure 4. Finally, the dynamics of $\widetilde{R}$ only depends on the genes of the two symmetric strongly connected components presented above and the necessary and sufficient conditions to apply the theorems 1 and 2 are respected by the Mendoza network, which explains why its sequential dynamics leads only to fixed points and why its parallel dynamics leads to the same fixed points and to limit cycles of period 2 .

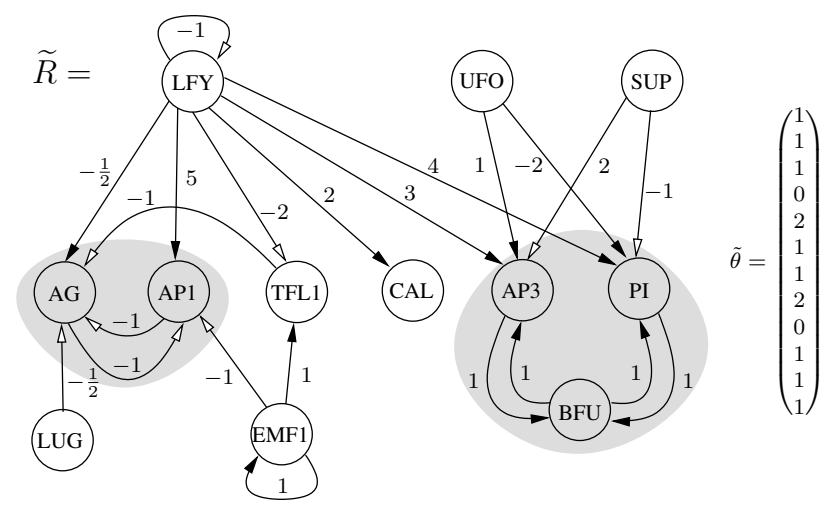

Fig. 4. Genetic regulatory network with two symmetric strongly connected components, whose dynamics has the same attractors as the Mendoza network. The threshold vector is ordered as in Figure 3.

\section{Perspectives}

We have shown that non-linear artificial regulatory networks on $\mathbb{Z}^{2}$ are highly dependent on boundary conditions under certain conditions. A further study could concern regular networks (progressively) perturbed by removing a certain (increasing) percentage $\epsilon$ of their interactions: if this architectural "surgery" is done at random and fixed before studying the dynamics of the resulting network, such as in [5], we could expect that, among the simulated non regular networks, some are similar to biological regulatory networks for which we have already shown the presence of a high dependence on boundary states [22]. Furthermore, it would be of interest to obtain the value of $\epsilon$ for which no phase transitions can be found.

The notion of symmetrisation developed in this work would also require further studies. First, it allows to go to and from between the theoretical framework and the applied framework. On this point, it would be of interest to show what already known real biological networks get this property or others and, thus, for which it would exist a natural bridge between these different abstraction levels. Moreover, on the study presented here about the Arabidopsis thaliana floral 
morphogenesis regulatory network, the fact that it is symmetric is important because for symmetric threshold Boolean automata networks, one may define an energy function on the dynamics and, then, attractors (fixed points and limit cycles) are energy's local minima. This will give an enlarged regard on this floral morphogenesis model.

\section{Acknowledgements}

This work has been supported in part by Eric Goles project Fondecyt 1070022; BASAL CMM and PROJECT STIC_SUD.

\section{References}

1. Albert, R., Barabási, A.L.: Statistical Mechanics of Complex Networks. Reviews of Modern Physics 74 (2002) 47-97

2. Kitano, H.: Biological robustness. Nature Reviews Genetics 5 (2004) 826-837

3. Elena, A., Demongeot, J.: Interaction Motifs in Regulatory Networks and Structural Robustness. In: Proceedings of the Second International Conference on Complex, Intelligent and Software Intensive Systems, IEEE Press (2008) 682-686

4. Goles, E., Salinas, L.: Comparison between Parallel and Serial Dynamics of Boolean Networks. Theoretical Computer Science 396 (2008) 247-253

5. Fates, N., Morvan, M.: Perturbing the Topology of the Game of Life Increases Its Robustness to Asynchrony. In: Cellular Automata. Volume 3305 of LNCS., Springer (2004) 111-120

6. Demongeot, J., Sené, S.: Boundary Conditions and Phase Transitions in Neural Networks. Simulation Results. Neural Networks 21 (2008) 962-970

7. Sené, S.: Influence des conditions de bords dans les réseaux d'automates booléens à seuil et application à la biologie. PhD thesis, Université Joseph Fourier de Grenoble (2008)

8. Jopling, C.L., Yi, M., Lancaster, A.M., Lemon, S.M., Sarnow, P.: Modulation of Hepatitis C Virus RNA Abundance by a Liver-Specific MicroRNA. Science 309 (2005) 1577-1581

9. Mendoza, L., Alvarez-Buylla, E.R.: Dynamics of the Genetic Regulatory Network for Arabidopsis thaliana Flower Morphogenesis. Journal of Theoretical Biology 193 (1998) 307-319

10. de Duve, C.: Life Evolving. Oxford University Press (2002)

11. Eigen, M., Gardiner, W., Schuster, P., Winkler-Oswatitsch, R.: The Origin of Genetic Information. Scientific American 244 (1981) 88-92

12. Gardner, M.: Mathematical Games. The Fantastic Combinations of John Conway's New Solitaire Game "Life". Scientific American 223 (1970) 20-123

13. Hopfield, J.J.: Neural Networks and Physical Systems with Emergent Collective Computational Abilities. Proceedings of the National Academy of Science of the USA 79 (1982) 2554-2558

14. McCulloch, W.S., Pitts, W.: A Logical Calculus of the Ideas Immanent in Nervous Activity. Journal of Mathematical Biology 5 (1943) 115-133

15. Cinquin, O., Demongeot, J.: Positive and Negative Feedback: Striking a Balance Between Necessary Antagonists. Journal of Theoretical Biology 216 (2002) 229241 
16. Kaufman, M., Soulé, C., Thomas, R.: A New Necessary Condition on Interaction Graphs for Multistationarity. Journal of Theoretical Biology 248 (2007) 675-685

17. Demongeot, J., Jézéquel, C., Sené, S.: Boundary Conditions and Phase Transitions in Neural Networks. Theoretical Results. Neural Networks 21 (2008) 971-979

18. Dobrushin, R.L.: The Problem of Uniqueness of a Gibbsian Random Field and the Problem of Phase Transitions. Functional Analysis and Its Applications (1968)

19. Goles, E., Martinez, S.: Neural and Automata Networks. Kluwer Academic Publishers (1990)

20. Goles, E., Olivos, J.: Comportement périodique des fonctions seuils binaires et applications. Discrete Applied Mathematics 3 (1981) 93-105

21. Fogelman-Soulie, F., Goles, E., Weisbuch, G.: Transient Length in Sequential Iteration of Threshold Functions. Discrete Applied Mathematics 6 (1983) 95-98

22. Ben Amor, H., Demongeot, J., Sené, S.: Structural Sensitivity of Neural and Genetic Networks. In: MICAI. Volume 5317 of Lecture Notes in Computer Science., Springer (2008) 973-986 